Influencia de la migración en los precios de la vivienda en los municipios de Cundinamarca (Colombia)

\author{
Hernán Enríquez
}


Lecturas de Economía, 79 (julio-diciembre 2013), pp. 171-197

Hernán Enriquez.

Influencia de la migración en los precios de la vivienda en los municipios de Cundinamarca (Colombia)

Resumen: El articulo evalúa la relación de interdependencia que existe entre el valor de la vivienda urbana y el crecimiento poblacional vía migración, bajo un modelo de mercado inmobiliario regional en Cundinamarca (Colombia). Se tiene en cuenta la accesibilidad a Bogotá en la determinación conjunta de estas variables, con el fin de determinar bajo qué condiciones se ajusta el mercado controlando por factores espaciales. Se estima un sistema de ecuaciones para los precios de la vivienda urbana y la tasa neta de migración para 116 municipios. El principal resultado indica que la mayor atracción de población de un municipio influye negativamente en el valor de la vivienda urbana. Como conclusión, se tiene que bajo la noción de interdependencia precios-migración mediada por el nivel de cercania a la capital, se configura un mercado inmobiliario regional que no se vincula entre municipios y que ofrece bajos niveles de amenidades a la población en cada localización.

Palabras clave: migración, mercado inmobiliario, amenidades.

Clasificación JEL: C31, J61, R31

Influence of migration on housing prices in the municipalities of Cundinamarca (Colombia)

Abstract: This paper evaluates the interdepent relation between urban housing values and population growth via migration, based on a regional housing market model in Cundinamarca (Colombia). The influence of accessibility to Bogota is taken into account in the joint determination of these variables with the aim to determine under what conditions the market adjusts, controlling for spatial factors. Using data from 116 municipalities, a system of equations is estimated for urban housing prices and net migration rate. The main result shows that a municipality's higher attraction of people has a negative influence on urban housing values. As a conclusion, under interdependency between housing prices and migration controlled for accessibility to the capital city, the regional housing market is not linked across municipalities and offers poor amenity levels to people in each location.

Keywords: Migration, housing market, amenities.

JEL classification: $C 31, \mathrm{~J} 61, \mathrm{R} 31$

Influence des migrations sur le prix du logement dans les communes de la région de Cundinamarca (Colombie)

Résumé: Cet article examine l'interdépendance entre la valeur du logement urbain et la croissance de la population migrante dans un modèle de marché immobilier régional appliqué à la région de Cundinamarca (Colombie). Nous prenons en compte l'accessibilité à la ville de Bogotà dans la détermination conjointe des variables, afin de déterminer dans quelles conditions d'ajustement du marché immobilier, tout en contrôlant par les facteurs spatiaux. On estime ainsi un système d'équations aussi bien pour les prix des logements urbains que pour le taux net de migration pour les 116 communes de la région de Cundinamarca. Les résultats montrent que plus il y a des nouveaux arrivants dans une commune, plus diminue la valeur du logement urbain. En conclusion, étant donné le concept d'interdépendance prix-migration mis en rapport avec le niveau de proximité avec la capitale, nous montrons qu'un marché immobilier régional n'est pas lié entre les différentes communes et qu'il offre des possibilités réduites à la population dans chaque emplacement.

Mots-clés: migration, marché du logement, équipements collectifs

Classification JEL: C31, J61, R31 


\title{
Influencia de la migración en los precios de la vivienda en los municipios de Cundinamarca (Colombia) ${ }^{1}$
}

\author{
Hernán Enríquez* \\ -Introducción. -I. Marco Teórico. -II. Datos. -III. Estimaciones y resultados. \\ - Conclusiones. -Bibliografía. -Anexos. \\ doi: 10.17533/udea.le.n79a6
}

Primera versión recibida el 26 de febrero de 2013; versión final aceptada el 4 de mayo de 2013

\section{Introducción}

Una nueva dinámica comienza a ser evidente en los municipios de Cundinamarca como resultado de las restricciones de habitabilidad impuestas por el mercado a una buena parte de la población. La relocalización de personas de Bogotá en los municipios vecinos, donde existe un mayor acceso a suelo urbanizable y la continua migración a estos desde otros municipios del país, refuerzan la atractividad del asentamiento de población en las cercanías de la capital y fortalecen el surgimiento de las ciudades intermedias con el consiguiente reforzamiento de la metropolización implícita que existe en la región.

El efecto de la aglomeración que había permitido a los nuevos habitantes de la ciudad acceder a fuentes de empleo y lugares habitables ha venido agotándose lentamente. La escasez de suelo y la imposibilidad de expansión

1 Este trabajo se realiza para el año 2005. Debido al uso de información censal no es posible trabajar con una fecha más reciente.

* Hernán Dario Enríquez. Sierra: Docente e investigador en áreas de la economía regional y métodos cuantitativos. Actualmente se desempeña como investigador del GIPE de la Universidad Sergio Arboleda. Dirección postal: Carrera 15 No 74-70, piso 3, Bogotá. Dirección electrónica: hernan.enriquez@usa.edu.co. 
Hernán Enríquez: Influencia de la migración en los precios de la vivienda...

del área urbana se presentan como uno de los principales determinantes de este fenómeno, que tiene su principal señal en los elevados precios de la vivienda que se observan para distintos estratos socioeconómicos al interior de la ciudad.

Como resultado de esta dinámica, se tiene un creciente flujo de personas que se reubican en los municipios en las cercanías de la capital con el fin de aprovechar las ventajas que ofrece la ciudad, asumiendo menores costos de vida. De esta manera, la migración toma una renovada importancia, puesto que define la configuración de los municipios bajo la idea de las condiciones locales que estos ofrezcan para el asentamiento de la población y las posibilidades que tengan los nuevos residentes para acceder a empleo e ingresos en el propio municipio o en Bogotá.

Si bien no existe un referente al tema de la migración a nivel de Cundinamarca, la literatura de migración interregional ha analizado los cambios de estructura que ha sufrido este componente de crecimiento poblacional a nivel nacional. Diferentes estudios sobre migración interdepartamental coinciden en que los principales factores que influyen en el fenómeno de la migración son los ingresos, mayor acceso al sistema educativo y de salud, y las oportunidades de vinculación al mercado laboral ${ }^{2}$, entre otros, se destacan los trabajos de Murad (2003), Granados (2010), Guarnizo (2006), Jaramillo (1999), y Martínez (2000).

Romero (2010) destaca que en Colombia la población migra de las zonas más rezagadas a las zonas más desarrolladas económicamente, lo que en la mayoría de los casos lleva implícito un proceso de concentración del capital humano debido a que la población con mayor grado educativo es la que presenta mayor tendencia a emigrar hacia poblaciones más prósperas. Esta posición es compartida por Fields (1980), quien sostiene que la migración interna en Colombia está estrechamente relacionada con las oportunidades que ofrecen regiones más desarrolladas del país, en términos de ingresos

2 DANE (2003) sostiene que estos factores son determinantes desde 2003 bajo una dinámica de desplazamiento interdepartamental entre áreas urbanas y cabeceras departamentales, más que un movimiento de largas distancias que era como se venía presentando el fenómeno. 
y empleo, y que la rápida urbanización de las principales ciudades se puede explicar, en gran medida, por la dinámica de la migración.

Galvis (2002) establece, mediante la estimación de un modelo gravitacional, que la dinámica de migración interna también responde a factores de ámbito espacial, como las características del lugar de origen y de destino. El autor precisa que las principales variables relacionadas con el proceso migratorio son la distancia y la dirección del desplazamiento. Utilizando como variable la distancia recorrida al emigrar, el autor determina que la población presenta una tendencia migratoria hacia sitios centrales del país.

Los estudios sobre migración en Colombia se han detenido únicamente en el proceso de desplazamiento de la población, obviando los efectos locales que este pueda tener en cada una de sus dimensiones. Específicamente, resulta interesante analizar cambios en patrones locales a partir de la entrada o salida de personas a un municipio determinado. Para poder indagar sobre este tipo de procesos, se acude al valor de las viviendas como una aproximación al sistema de preferencias urbanas de los individuos - controlando por el nivel de ingresos- que, además, puede representar los cambios en los patrones de utilidad de las personas de una localización.

El propósito de este artículo es evaluar cómo la migración a Cundinamarca ha afectado los precios de la vivienda en los municipios, contando con el vínculo que se tiene por proximidad con Bogotá. Con base en las teorías clásicas de migración, junto a algunos postulados de la economía urbana, se estima un modelo econométrico que permite endogeneizar el cambio poblacional relacionado a la migración en cada municipio del departamento y calcular el efecto de esta sobre el valor medio de la vivienda en las cabeceras municipales. Este esquema de análisis permite inferir cómo reacciona el mercado ante la llegada de la población migrante y qué nivel de servicios urbanos está dispuesto a ofrecer para satisfacer sus preferencias.

Las estimaciones indican que, en primer lugar, no existe correlación espacial ni para el valor de las viviendas ni para la migración en el departamento. En segundo lugar, tanto para la migración como para el valor de los inmuebles, la accesibilidad a Bogotá es relevante, pero no es el principal determinante de su cambio. Por último, se tiene que el valor de la vivienda se ve 
Hernán Enríquez: Influencia de la migración en los precios de la vivienda...

negativamente afectado por la llegada de personas, aunque se muestra poco sensible a este fenómeno.

El artículo se divide en cuatro secciones. En la primera, se expone el modelo que relaciona de manera endógena a las variables de interés. Los datos utilizados y las relaciones de dependencia espacial se describen en la segunda sección. En la tercera sección se estima un modelo de ecuaciones simultáneas y se discuten sus principales resultados. Por último, se exponen las conclusiones más relevantes del trabajo.

\section{Marco Teórico}

La relocalización de la población en el espacio ha sido ampliamente estudiada desde distintas disciplinas y con distintos enfoques ${ }^{3}$. La migración es entendida como una fuente importante de generación de actividad económica y riqueza local, regional y nacional, desde aproximaciones al mercado laboral (Sjaastad, 1962; Todaro, 1969; Harris \& Todaro, 1970); Greenwood, Hunt, Rickman \& Treyz, 1991), las condiciones para la movilidad (Mincer, 1978), las características locales expresadas en amenidades (Graves \& Linneman, 1979; Graves, 1976), o el crecimiento económico (Kuztnets, 1964), entre otros.

La dinámica migratoria se ha analizado con un amplio uso de la contrastación empírica de los principales determinantes asociados a los enfoques arriba mencionados. Siguiendo a Greenwood (1985) y Waltert (2010), los factores que inciden en el movimiento de población se pueden clasificar en: i. Condiciones del individuo y su familia: niveles de destreza, edad, educación, estado civil, número de hijos, etc.; ii. Condiciones de la producción: productividad factorial, especialización productiva, tecnología; iii. Condiciones de las localizaciones: amenidades creadas, bienes y servicios públicos, instituciones, mercado de vivienda, criminalidad, etc.; y iv. Factores ambientales: amenidades propias, factores contaminantes, cercanía a bienes de carácter natural, etc.

3 Por la naturaleza del trabajo, solo se realizará una breve descripción de los principales postulados teóricos relacionados con el estudio de la migración interna por motivos económicos. 
Controlando por un conjunto de determinantes se pueden evaluar los efectos que tiene la dinámica migratoria en los aspectos locales, los cuales son representados en términos de accesibilidad a bienes y servicios, y a las condiciones mismas del mercado laboral en los sitios de origen y destino de los migrantes. El reflejo de la situación migratoria se presenta en el comportamiento de los índices de precios, las rentas de las viviendas y la distribución de los salarios.

El continuo movimiento de población supone un constante ajuste regional en los niveles de precios y salarios para cada localización. El flujo migratorio se detendrá cuando se alcance el equilibrio espacial, en el que para distintos niveles de precios, salarios y rentas por localización, un individuo obtiene una utilidad constante a través del espacio. Esto se logra por medio de los efectos compensatorios a estas señales y que son inherentes a cada ciudad (Graves 1983).

El enfoque tratado aquí se relaciona con el efecto de la migración en el mercado de vivienda. En este campo, los aportes teóricos de Graves (1980), Graves \& Linemann (1979), Greenwood (1985), entre otros, han marcado la línea de análisis sobre los aspectos generales que enmarcan el proceso de migración y determinación de los precios de las viviendas. Si bien en un principio la relación entre estas dos variables puede ser obvia, el cálculo del efecto no lo es, debido a la simultaneidad en la que, en la mayoría de las veces, se presentan, y al efecto dinámico que se da entre ellas (Jeanty, Partridge \& Irwin, 2010).

La simultaneidad entre las variables mencionadas es la que conduce al interrogante sobre el efecto real de la migración sobre los precios de las viviendas y cómo los determinantes del flujo definitivo de personas entre localizaciones pueda influir, positiva o negativamente, en el valor de las viviendas urbanas.

La importancia de la migración en el contexto de un mercado inmobiliario se entiende si es tenida en cuenta como el componente del crecimiento poblacional que permite incorporar las expectativas de niveles más altos de población en el futuro, la cual requerirá de viviendas para habitar bajo un sistema de preferencias individuales. El ajuste de dichas expectativas conducirá a una variación de los precios de las viviendas en una localidad específica (Potepan, 1994). 
Hernán Enríquez: Influencia de la migración en los precios de la vivienda...

Con el fin de analizar la simultaneidad entre precios de vivienda y la migración poblacional desde una perspectiva regional, Potepan (1994) incluye en un modelo de mercado de vivienda al componente migratorio. Desde una óptica de oferta y demanda de vivienda que incluye el crecimiento de la población, características geográficas y el nivel local de amenidades, los precios de las viviendas y la dinámica migratoria estarán determinados por las ecuaciones 1 a 3 (Potepan, 1994, p. 82):

$$
\begin{aligned}
& P H_{i}=f\left(I M_{i}, O M_{i}, X h_{i}\right) \\
& I M_{i}=f\left(P H_{i}, X m_{i}\right) \\
& O M_{i}=f\left(P H_{i}, X m_{i}\right)
\end{aligned}
$$

Se tiene un sistema de ecuaciones simultáneas que define para la localización $i$ la determinación de los precios de vivienda $P H$, como una función de los niveles de inmigración $I M$, emigración $O M$ y un vector de variables $X h$ que resumen las características propias del mercado, como el ingreso medio de los hogares, el nivel de impuestos, precios del suelo disponible para construir, características topográficas, niveles de amenidades y características regionales, entre otras.

En las ecuaciones 2 y 3, los niveles de inmigración y emigración se encuentran determinados por los precios de las viviendas y un vector de variables Xm que definen cada flujo de población como las descritas en la clasificación mencionada arriba. La inmigración aumenta los precios de las viviendas, mientras que los precios de las viviendas, dependiendo de determinantes de productividad y amenidades, pueden incrementar la inmigración, la emigración o terminar en un intercambio de población entrante y saliente.

\section{Datos}

Para evaluar el efecto de la migración sobre los precios de la vivienda, se recopilan datos de las variables descritas en la tabla del anexo 1. La información es de carácter municipal, recopilada del censo de 2005, el anuario esta- 
dístico de Cundinamarca, Redatam y el Instituto Geográfico Agustín Codazzi (IGAC). Se utilizan 116 municipios sin incluir al distrito capital.

Según el Censo de 2005, la región Bogotá-Cundinamarca concentra el $21 \%$ del total de la población, siendo Bogotá el principal centro urbano del país con un número de habitantes que superaba, para esa fecha, los seis millones de habitantes. Un fuerte poder de atracción de población, que por distintas circunstancias se asentó en su perímetro urbano mantiene a la ciudad como la de mayor tamaño y, al mismo tiempo, la que lidera la primacía poblacional en el país (CIDS, 2007).

Los indicadores de migrantes netos para el departamento muestran un cambio en el flujo de población en Cundinamarca. Para la segunda mitad de la década de los ochenta se expulsaban en el departamento a 33,8 personas de cada mil habitantes, mientras que en la primera mitad de la década del 2000 se pasa a atraer a 33,6 personas por mil habitantes (DANE, sf.). Municipios ubicados en las cercanías de la ciudad son los que más han capturado el flujo de nuevos pobladores -e.g. Soacha, Mosquera, Chía y La Calera-, aunque la dinámica migratoria se extiende a todo el departamento.

Los precios de la vivienda urbana en Cundinamarca han tomado una dimensión diferente respecto de la actividad de cada uno de los municipios y la cercanía a Bogotá, como se evidencia en el Mapa 1. En primer lugar, en aquellos sitios donde predomina el turismo y las viviendas de descanso, los precios son considerablemente más altos. Este es el caso de la concentración de altos niveles de precios en los municipios de Sasaima, Villeta, Apulo, Anapoima, La Mesa y Ricaurte, al occidente del departamento.

Por otra parte, la cercanía a la capital del país muestra contrastes respecto a la formación de precios, distinguiéndose el comportamiento de precios altos al norte de la ciudad en municipios como Chia, Zipaquirá, Cajicá y Sopó, los que se encuentran en conexión por la autopista norte. Sin embargo, municipios como Funza, Mosquera, Facatativá, al occidente y Soacha al sur de Bogotá, que guardan una mayor cercanía a la ciudad, evidencian precios más bajos. Esta dinámica es coherente con la que se observa en la capital, por lo que se están prolongando a las cercanías los vectores de precios observados en los ejes norte y suroccidental de la ciudad. 
Hernán Enríquez: Influencia de la migración en los precios de la vivienda...

Mapa 1. Avalúo medio de las viviendas urbanas. Millones de pesos de 2005

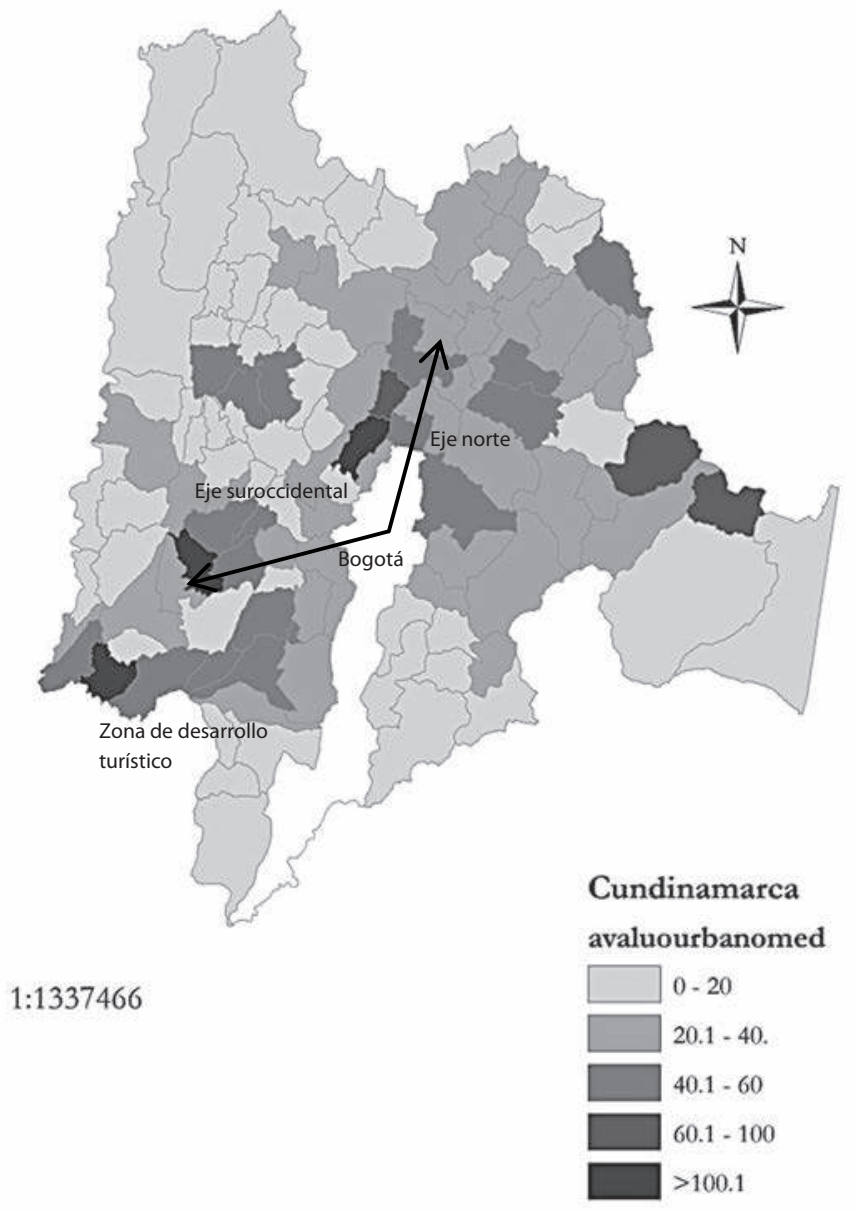

Fuente: elaboración propia a partir de información de IGAC.

El Mapa 2 ilustra el fenómeno de migración neta municipal en el departamento, que resulta de la diferencia entre las personas que se ubicaron menos las que abandonaron cada municipio. Se puede observar que en general el departamento atrae población. La mayor atractividad se concentra al suroccidente, alrededor de dos áreas: la primera comprende los municipios de Soacha, Sibaté, Fusagasugá, Arbelaez y Pandi, los que guardan una relación de cercanía con 
la capital'; ; la segunda se conforma por los municipios de Anapoima, Apulo, Tocaima, Ricaurte y Nariño. Algunos de estos últimos evidencian altos precios de las viviendas como se muestra con el Mapa $1 .^{5}$

Mapa 2. Migración neta. Cundinamarca. 2005

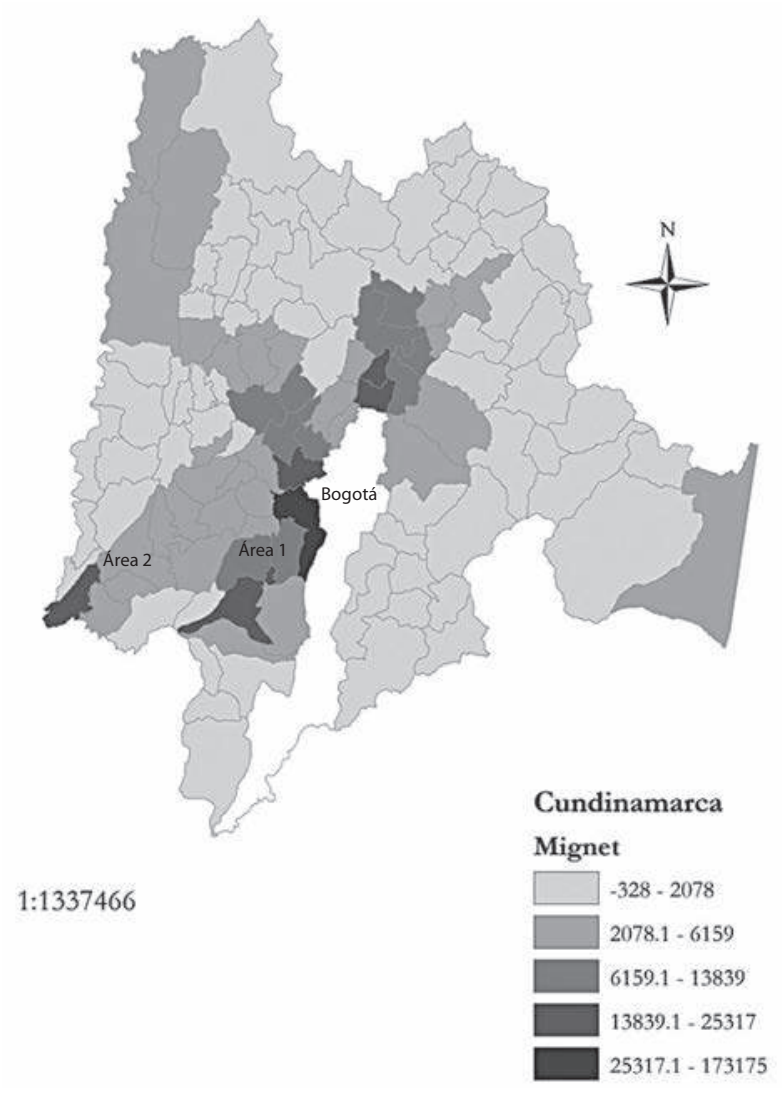

Fuente: elaboración propia a partir de información de Redatam.

4 No se cuenta con información desagregada para caracterizar a los migrantes en el nivel municipal, por lo que no es posible inferir las características personales de la población que se movilizó en el periodo de análisis.

5 En el anexo 3 se muestran las relaciones entre migración y precios para los municipios que más atraen población y los que más expulsan. 
Hernán Enríquez: Influencia de la migración en los precios de la vivienda...

El análisis preliminar sugiere dos aspectos que desde el punto de vista espacial denotan interés para evaluar la dinámica, tanto de precios de vivienda como de la migración, en el departamento. En primer lugar, se puede pensar que existe algún nivel de dependencia espacial para las variables que están siendo analizadas; esto puede indicar que los precios y la migración observados en un municipio dependen de lo que ocurre en las localizaciones vecinas a este. En segundo lugar, el análisis exploratorio espacial muestra que, entre más positiva es la tasa de migración neta, menores son los precios de las viviendas.

Para el caso de la dependencia espacial, tanto de precios de vivienda como de la tasa de migración neta, se calcula el índice de Moran, el cual determina estadísticamente si existe influencia de vecindades en la realización de una variable para una localización específica. La Tabla 1 resume los resultados del test para las variables, utilizando una matriz de pesos convencional, bajo criterio de contigüidad tipo reina ${ }^{6}$.

Tabla 1. Test de Moran

\begin{tabular}{ccc}
\hline & logvivurbmed & migneta \\
\hline Desviación estándar estadístico Moran I & $-0,184$ & $-0,7$ \\
P-valor & 0,573 & 0,758 \\
Estadístico Moran I & $-0,0195$ & $-0,0503$ \\
Valor esperado & $-0,0087$ & $-0,0087$ \\
Varianza & 0,0035 & 0,0035 \\
\hline
\end{tabular}

Notas: Hipotesis nula: No autocorrelación espacial logvivurbmed: logaritmo del avalúo medio de las viviendas urbanas migneta: Tasa de migración neta por $10 \mathrm{mil} \mathrm{hab.}$

Fuente: elaboración propia

Los resultados de la prueba indican que no existe una relación espacial en los precios medios de las viviendas ni en la tasa de migración neta. Con

6 La matriz de pesos o de vecindad se usa para cuantificar las relaciones de contigüidad entre unidades espaciales que pertenecen a una región. Las matrices convencionales se construyen de manera binaria en donde se indica en las filas, con el valor uno, cuando una localización es vecina de otra a partir de alguno de los criterios de vecindad utilizados. Los pesos provienen de la estandarización de la matriz para que cada una de sus filas sume la unidad. Ver más detallado en LeSage \& Pace (2009). 
este resultado se puede afirmar que los precios observados en un municipio no dependen de los observados en sus vecinos. De la misma manera, la atracción o expulsión de población de un municipio no es influenciada por lo que ocurra con el movimiento poblacional de sus vecinos ${ }^{7}$.

Por último, para precios y para migración, la cercanía a Bogotá es un factor que influye en su determinación -como se podría intuir-. Sin embargo, como se mostrará en la siguiente sección, aunque no es el único elemento explicativo, es claro que las interacciones con la capital del país influyen en alguna medida el comportamiento observado en todo el departamento para las variables de interés de este trabajo.

\section{Estimación y resultados}

La estrategia metodológica para la especificación y estimación del efecto de la migración sobre los precios de la vivienda se caracteriza por la relación de simultaneidad entre estas variables, tal y como se establece en el trabajo de Potepan (1994), y por la inexistencia de una relación de dependencia espacial, como se mostró en la sección anterior ${ }^{8}$. De esta manera, se especifica un modelo de ecuaciones simultáneas -como en Bischoff, 2012; Greenwood, 1975; Jeanty et al., 2010; Potepan, 1994, 1996) - que se describe en las ecuaciones 4 y 5.

$$
\begin{aligned}
& \operatorname{lnpvu}_{i}=\operatorname{\varphi migneta}_{i}+X_{1 i} \beta_{1}+u_{1 i} \\
& \text { migneta }_{i}=\varnothing \operatorname{lnpvu_{i}}+X_{2 i} \beta_{2}+u_{2 i}
\end{aligned}
$$

Las variables dependientes del sistema son el logaritmo del precio medio de la vivienda urbana lnpun y la tasa de migración neta por diez mil habitantes

7 El lector debe notar que la prueba se realiza solo para los municipios del departamento y que sus resultados no se pueden extender a la influencia que pueda tener Bogotá en cada uno de ellos. Esta última será controlada e interpretada en la siguiente sección.

8 Como no existe dependencia espacial de las variables endógenas del sistema, no es necesario acudir a una especificación econométrica que incluya rezago espacial de dichas variables. Pruebas de Moran por Máxima verosimilitud realizadas a los residuos del modelo final estimado confirman que no se debe incluir control por vecindad, ni de las variables dependientes, ni de los errores. Ver Tabla 2. 
Hernán Enríquez: Influencia de la migración en los precios de la vivienda...

, para cada uno de los municipios migneta del departamento. Bajo la hipótesis de simultaneidad se incluye cada una de estas variables como determinante de la otra en cada ecuación, acompañadas de su respectivo coeficiente.

Para controlar las condiciones del sistema se incluye en cada ecuación una matriz de determinantes de cada variable dependiente. En la matriz $X_{1}$ se incluye el logaritmo de ingreso per cápita como aproximación al ingreso de los hogares que sugiere el modelo de Potepan (1994). Adicionalmente, se tienen en cuenta variables que ejercen presión sobre el mercado de vivienda -como especifican Bischoff (2012), y Jeanty et al. (2010)-, como son el logaritmo de la densidad poblacional por kilómetro cuadrado y el logaritmo de los ingresos municipales por cuenta del impuesto predial.

Con el objeto de incluir amenidades locales relacionadas con el mercado de vivienda, se incluye como variable independiente a la proporción del suelo municipal que es rural, ya que puede mostrar la capacidad municipal de extender su centro urbano y, al mismo tiempo, la presión sobre los recursos naturales y bienes ambientales existentes en el suelo rural (Chen et al., 2009; Waltert \& Schläpfer, 2010). Por su parte, variables como la tasa de lesiones personales por cada cien mil habitantes y una dummy de piso térmico frío son incluidas para controlar condiciones institucionales y geográficas de cada municipio.

Siguiendo a Graves (1980) Greenwood (1985) y a Jeanty et al. (2010), la matriz $x_{2}$ contiene las variables: logaritmo del ingreso per cápita, tasa de desempleo municipal, el logaritmo del número promedio de integrantes del hogar y de la población menor de 14 años, así como el porcentaje de personas con nivel de escolaridad bachillerato. Igualmente, se incluye la variable dummy para identificar los municipios situados en piso térmico frio y la tasa de lesiones personales como una proxy de las condiciones de criminalidad e instituciones. En el caso de las variables incluidas en esta matriz, se desea observar cómo estos factores conducen a que un municipio sea más o menos atractivo para la población migrante.

Para las dos ecuaciones se incluye una variable que permite capturar la interacción entre cada municipio y la ciudad de Bogotá. Para este caso, se construye un indicador de proximidad espacial que tenga en cuenta, en adición a la cercanía física, la accesibilidad real a la ciudad. Esto debido a la geografía 
del departamento, la cual permite tener casos en los que municipios más cercanos a la ciudad tengan un mayor tiempo de viaje a ésta, que otros que se encuentran más alejados.

La medida de accesibilidad se obtiene como el coeficiente entre la distancia en carretera de cada cabecera municipal al centro de Bogotá -medida en kilómetros-, ajustada por el cuadrado del tiempo estimado de viaje -en horas-. La ecuación 6 muestra la forma de calcular el índice de accesibilidad?

$$
\text { acces }_{i}=\frac{d B t a_{i}}{t B t a_{i}^{2}}
$$

La medida de accesibilidad a Bogotá es estandarizada para facilitar la comparación entre municipios. En este caso, un valor próximo a cien reflejará mejores condiciones de acceso a la capital relativa a los demás municipios considerados.

El sistema comprendido por las ecuaciones 4 y 5 se estima por el método de Mínimos Cuadrados en tres etapas, el cual es usado para obtener los parámetros de las ecuaciones simultáneamente y mejorar la eficiencia de los estimadores $^{10}$, evitando la inconsistencia generada por la posible correlación de variables con los residuos de las ecuaciones del sistema (Greene, 2012). Los resultados se muestran en la Tabla $2^{11}$.

El principal resultado obtenido de la estimación del modelo estructural indica que, a pesar de lo que se espera en la teoría económica, para los municipios de Cundinamarca los incrementos en la tasa de migración neta conducen a un descenso en los precios de las viviendas urbanas. La reducción del valor es del orden del $0,39 \%$ por cada migrante que llegue al centro urbano -ajustado por cada 10 mil habitantes-.

9 Los datos para el cálculo del índice fueron obtenidos por medio de Google Maps ${ }^{\circledR}$.

$10 \mathrm{El}$ funcionamiento del método consiste en una estimación por mínimos cuadrados generalizados factibles de una regresión en dos etapas, estimando previamente la matriz de varianzas y covarianzas de los residuos del sistema, con el fin de asegurar la eficiencia de los estimadores. Ejemplos de este tipo de estimación se encuentran en Bischoff (2012) y en Poudyal, Hodges \& Cordell (2008).

11 Para obtener propiedades estadísticas adecuadas para los residuales del sistema se incluyen controles sobre datos atípicos. Los coeficientes de esas variables dummy no se muestran en la tabla. Ver estadísticas descriptivas de las variables utilizadas en el anexo 2. 
Hernán Enríquez: Influencia de la migración en los precios de la vivienda...

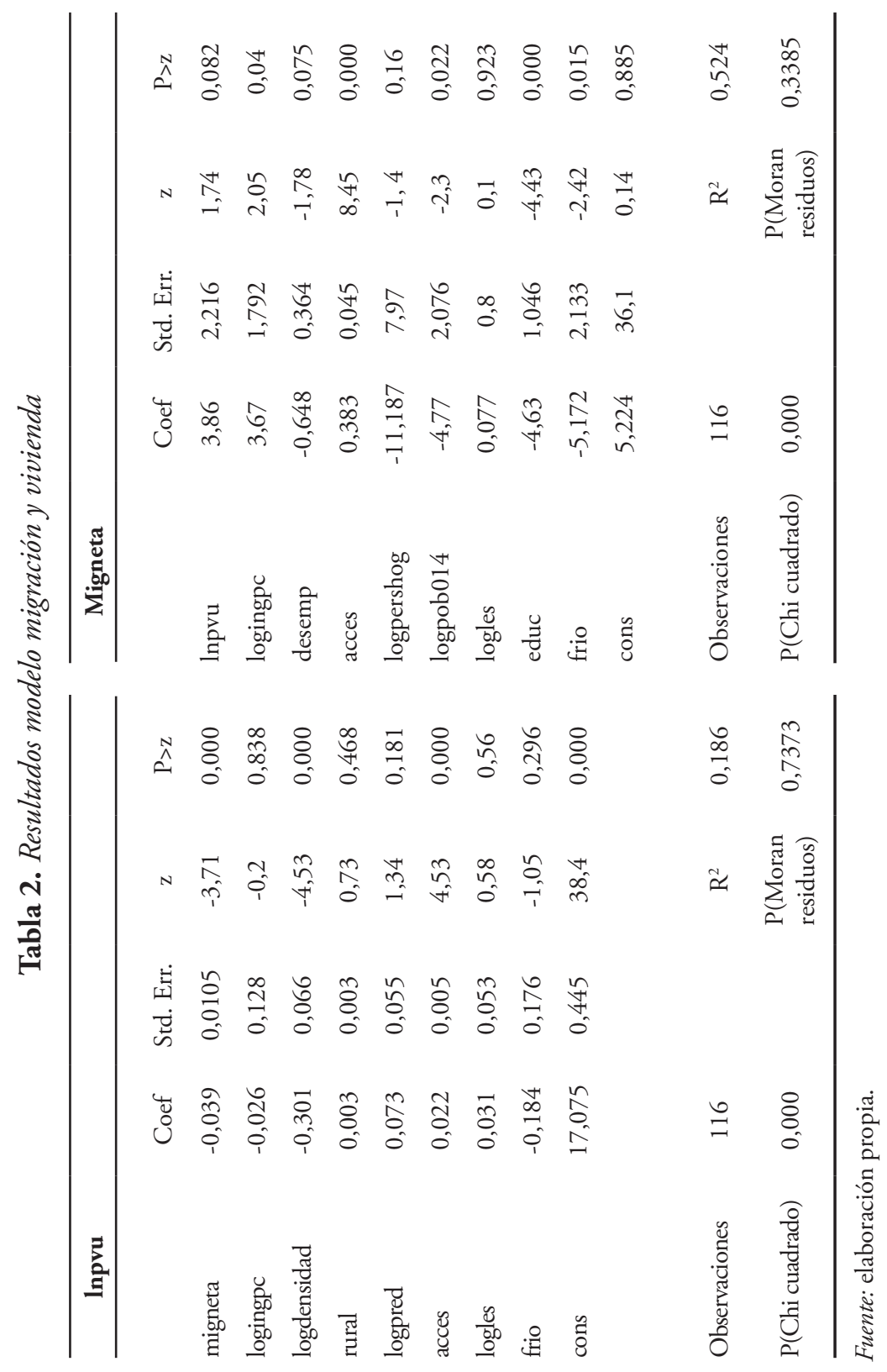


Las estimaciones obtenidas para la migración neta -ecuación 5- muestran que para aquellos municipios del departamento con altos niveles de ingreso se espera un mayor flujo de migrantes que busquen ubicarse allí. Este resultado sigue siendo coherente con las condiciones en las que se presenta la dinámica de atracción de migrantes y que fueron enunciadas a nivel interregional en Galvis (2002).

En cuanto al desempleo, no parece tomar mucha relevancia esta condición en la población a la hora de migrar, ya que, pese a que el coeficiente presenta el signo esperado, solo es significativo al 10\%. Este resultado indica que aun con restricciones a las fuentes de ingresos que impiden la llegada de población, existen otras características locales que previenen la salida de personas. Esta afirmación resulta coherente en un contexto en el que las amenidades locales son suficientes para contrarrestar el efecto negativo de ingresos bajos como lo sostiene Roback (1982).

Las variables demográficas incluidas en la ecuación dan cuenta de las condiciones de los hogares que promueven la salida de personas en un municipio. Se observa que, en la medida en que el tamaño de los hogares incremente, existe una mayor expulsión de personas -aunque estadísticamente no se tiene significancia-; la misma afirmación se presenta cuando se tiene un mayor número de personas jóvenes. En este sentido, se denota cómo las condiciones de los jóvenes los hacen más proclives a buscar reasentamiento en otras ciudades.

Analizando la magnitud de los efectos encontrados para la migración neta, el tamaño de la población joven y la educación presentan el mayor impacto estadísticamente significativo, en términos absolutos, lo que da lugar a pensar en que avances educativos no se materializan en el mercado laboral local sino, por el contrario, promueven la salida de población. Esto puede ser el resultado de las pocas oportunidades de encontrar salarios adecuados para una masa de población que busca mayores niveles educativos en un contexto de restringida demanda de empleos y baja acumulación de capital humano.

En cuanto al coeficiente que indica la conectividad con la capital del país, se tiene un efecto positivo de la accesibilidad a Bogotá para determinar la atracción migrante a un municipio. Efectivamente, a mayor posibilidad de 
Hernán Enríquez: Influencia de la migración en los precios de la vivienda...

acceso a la capital, el municipio atrae más población migrante - a razón de 3 inmigrantes por cada diez mil habitantes por unidad de aumento en la medida de accesibilidad-. Por su parte, las condiciones institucionales expresadas en la tasa de lesiones personales no tienen un efecto en las condiciones de migración.

Por último, se observa un patrón de salida de personas en los municipios del departamento a partir de las condiciones geográficas, las que en este caso permiten realizar un contraste entre los pisos térmicos existentes. Se tiene que, en promedio, expulsan más población aquellos municipios ubicados en pisos más altos. Aunque el sistema de ecuaciones no tiene el alcance necesario, se puede afirmar que en algunos municipios ubicados en pisos bajos se han encontrado oportunidades para sus pobladores en algunas actividades económicas, por el contrario, no se presenta una situación similar para los municipios que están en tierras más altas.

Los resultados obtenidos para los precios medios de la vivienda urbana -ecuación 4 muestran que el ingreso per cápita municipal no resulta ser un determinante para la determinación de los precios, contrario a lo que puede esperarse de esta variable por la importancia que tiene a la hora de tomar decisiones relacionadas a transacciones con un bien tan particular como lo es la vivienda.

Las condiciones urbanas reunidas en la densidad poblacional presentan un impacto negativo para los precios, que es incluso el de mayor efecto estimado sobre los precios. Este resultado es un indicativo de las características de las viviendas y de su habitabilidad cuando los municipios se encuentran altamente densificados. Al mismo tiempo, siguiendo a Graves (1976), se puede inferir que los municipios presentan déficits de amenidades entendidas como servicios públicos y privados, equipamientos y, en general, estructura urbana que presiona los precios a la baja. Todo esto sin contar posibles condiciones de hacinamiento de los hogares que no son controladas por el modelo.

Es de anotar que los impuestos sobre la propiedad no tienen incidencia sobre la determinación de los precios. La no significancia del coeficiente da lugar para pensar que el ajuste de los precios bajo las condiciones de mercado se realiza por las características propias de la vivienda y bajo el contexto 
mismo de los agentes que intervienen en la transacción, sin que medien distorsiones impositivas en la fijación del valor de las viviendas urbanas.

Las condiciones propias de los municipios, en términos ambientales e institucionales, no ejercen un efecto en la determinación de los precios de las viviendas urbanas. Si bien las variables proxy que se utilizan pueden resultar insuficientes para abordar plenamente el tema, los niveles de significancia que presentan, indican que en la fijación de precios no es relevante la oferta ambiental vista en la porción de suelo rural del municipio, ni las condiciones institucionales que se ofrezcan para la habitabilidad en el centro urbano. De la misma manera, los precios no cambian para municipios ubicados en distintos pisos térmicos, por lo que las amenidades naturales no cuentan de manera diferencial a la hora de determinar el valor de las viviendas urbanas.

Como en la migración, el coeficiente que mide la accesibilidad a Bogotá es positivo y significativo, demostrando la importancia relativa de las viviendas por su acceso a la ciudad. Para este caso se tiene que los precios incrementan en promedio $2,2 \%$ a razón de un aumento de una unidad del coeficiente.

\section{Conclusiones}

Los resultados obtenidos muestran un panorama diferente al esperado bajo el contexto del aprovisionamiento de viviendas para un grupo de población en continuo crecimiento. En primer lugar, dado que la migración afecta negativamente a los precios se prevé un efecto de reversión de las condiciones de mercado inmobiliario en el departamento. Es decir, a medida que la población migrante aumenta, la expectativa de reducción media de precios en el mediano plazo se va a acentuar.

La implicación de este resultado se puede evaluar desde la óptica individual y del papel de los gobiernos locales en un mercado regional que no se encuentra articulado espacialmente. En primer lugar, se tiene que las preferencias de la demanda se ven restringidas a la calidad de las viviendas que se pueden ofrecer a precios más bajos, por lo que posiblemente las condiciones de habitabilidad de las mismas se vean limitadas por reducción o eliminación 
Hernán Enríquez: Influencia de la migración en los precios de la vivienda...

de características de los inmuebles, como bien lo puede ser el área construida, materiales de menor calidad, menos espacios funcionales, etc.

En segundo lugar, se presenta un contraste entre el papel que juega cada gobierno local y la presión que puede recibir Bogotá frente a las condiciones de habitabilidad en los municipios. Por un lado, dado que las viviendas son de menor precio, el ente local debe proveer los servicios urbanos o amenidades creadas que no pudieron ofrecerse dentro de la compra de viviendas, esto implica acarrear mayores costos en la construcción de ciudad y en el aseguramiento de las condiciones para la vida digna de los ciudadanos.

Debido a la negativa reacción de los precios a la migración, los gobiernos municipales deben garantizar los efectos de compensación vía amenidades y lo que ello implica para los demás tipos de precios -salarios, rentas, de bienes y servicios-. Esto lleva a preguntarse hasta qué punto los municipios en los que la dinámica de reasentamiento de población es relativamente nueva, están preparados para la provisión de la estructura urbana que se requiere bajo expectativas de un crecimiento rápido de población.

Si bien esto representa un reto fiscal para los municipios del departamento, los resultados muestran que, para este caso, incrementos en el impuesto predial de los municipios pueden ser una medida que favorezca la provisión de amenidades sin que se vea afectado el mercado de vivienda. Este resultado permite equilibrar las condiciones del territorio en términos de habitabilidad sin que se distorsione el mercado.

Para el caso de Bogotá, por otro lado, se plantea la pregunta de cómo va a enfrentar el posible déficit de servicios urbanos en los municipios del departamento. Como se mostró, la accesibilidad a la ciudad es un factor clave para la determinación de la migración y los precios. Si bien la dinámica de los precios es conocida y evaluada desde los instrumentos de ordenamiento territorial, la presión de los migrantes asentados en otros municipios no ha sido contada.

Con un buen acceso a la ciudad es de esperar que las personas de otras localizaciones consuman en la capital los servicios públicos y privados que no están presentes en sus lugares de residencia y que pueden encontrar a gran y variada escala en la ciudad capital. Esto representa un desafío para la admi- 
nistración distrital y el departamento, puesto que se tendría que garantizar el acceso a la estructura urbana, no solo a residentes en la capital sino también a la creciente masa de población que los demande desde municipios cercanos. A su vez en el departamento se debe evitar que dichos municipios se transformen en ciudades dormitorio sin acceso a servicios urbanos.

\section{Referencias Bibliográficas}

BISCHOFF, Oliver (2012). "Explaining regional variation in equilibrium real estate prices and income", Journal of Housing Economics, Vol. 21, Issue 1, pp. 1-15.

Chen, Yong; Irwin, Elena \& Jayaprakash, Ciriyam (2009). "Dynamic modeling of environmental amenity-driven migration with ecological feedbacks", Ecological Economics, Vol. 68, Issue 10, pp. 2498-2510.

Centro de Investigación sobre Dinámica Social (CIDS) (2007) Ciudad, espacio y población: el proceso de urbanización en Colombia. Bogotá: UNFA.

Departamento Administrativo Nacional de Estadística (DANE) (Sf.). Indicadores demográficos de Cundinamarca. Recuperado de: http://www. dane.gov.co/files/investigaciones/poblacion/seriesp85_20/EstimacionesProyecciones1985_020.xls (31 de mayo de 2012).

FIElds, Gary (1980). "Migración permanente en Colombia”, Desarrolloy Sociedad, No. 3, pp. 109-115. Recuperado de: http://economia.uniandes.edu. co/layout/set/print/investigaciones_y_publicaciones/CEDC/Publicaciones/Revista_Desarrollo_y_Sociedad/Ediciones/revista_desarrollo_y_sociedas_no_3/Migracion_permanente_en_Colombia_Prueba_de_la_hipotesis_del_ingreso_esperesp (6 de agosto de 2012).

Galvis, Luis Armando (2002). "Determinantes de la migración interdepartamental en Colombia, 1988-1993", Documentos de trabajo sobre economía regional, No. 29, pp. 1-45. Recuperado de: http:/www.banrep.gov.co/es/ contenidos/publicacion/determinantes-migraci-n-interdepartamentalcolombia-1988-1993 (6 de agosto de 2012). 
Hernán Enríquez: Influencia de la migración en los precios de la vivienda...

GranAdos JimÉNEZ, Jennifer (2010). Las migraciones internas y su relación con el desarrollo en Colombia. Tesis para optar por el título de M.Sc en Desarrollo Rural. Facultad de Estudios Ambientales y Rurales, Pontificia Universidad Javeriana, Bogotá, Colombia. Recuperado de: http://www.javeriana.edu. co/biblos/tesis/eambientales/tesis27.pdf (10 de agosto de 2012).

Graves, Philip. (1976). "A Reexamination of Migration, Economic opportunity, and the Quality of Life", Journal of Regional Science, Vol. 16, Issue 1, pp. 107-112.

Graves, Philip (1980). "Migration and Climate", Journal of Regional Science, Vol. 20, No. 2, pp. 227-237.

Graves, Philip (1983). "Migration with a Composite Amenity: the Role of Rents”, Journal of Regional Science, Vol. 23, Issue 4, pp. 541-546.

Graves, Philip \& Lienneman, Peter (1979). "Human Migration: Theoretical and Empirical Results", Journal of Urban Economics, Vol. 6, Issue 1, pp. 383-404.

Greene, William H. (2012). Econometric Analysis (7th Ed.). New Jersey: Pearson Education.

Greenwood, Michael J. (1975). "A Simultaneous-Equations Model of Urban and Migration Growth", Journal of the American Statistical Association, Vol. 70, Issue 352, pp. 797-810.

Greenwood, Michael J. (1985). "Human Migration: Theory, models and empirical studies”, Journal of Regional Science, Vol. 25, No. 4, pp. 521-544.

Greenwood, Michael; Hunt, Gary; Rickman, Dan \& Treyz, George I. (1991). "Migration, Regional Equilibrium, and the Estimation of Compensating Differentials", The American Economic Review, Vol. 81, No. 5, pp. 1382-1390.

GuARnizo, Luis Eduardo (2006). "El Estado y la migración global colombiana.”, Migración y Desarrollo, No. 6, pp. 79-101. Recuperado de: http:// www.redalyc.org/articulo.oa?id=66000603\# (6 de agosto de 2012). 
Harris, John R. \& Todaro, Michael P. (1970). "Migration, unemployment and Development: A Two-Sector Analysis", The American Economic Review, Vol. 60, Issue 1, pp. 126-142.

Jaramillo, Samuel (1999). "Migraciones e interacción regional en Colombia, 1973-993”, Territorios, No. 1, pp. 95-117.

Jeanty, P. Wilner; Partridge, Mark \& Irwin, Elena (2010). "Estimation of a spatial simultaneous equation model of population migration and housing price dynamics", Regional Science and Urban Economics, Vol. 40, Issue 5, pp. 343-52.

Kuznets, Simon (1964). "Introduction: Population Redistribution migration and Economic Growth". In: H. Eldridge \& D.S Thomas (Eds.), Population redistribution and economic growth: United States, 1870-1950, Vol. 3. (pp. xxi-xxxiv). Philadelphia: The American Philisophical society.

LeSage, James \& Pace, Kelley (2009). Introduction to Spatial Econometrics. Boca Raton: Taylor \& Francis group.

Martínez Gómez, Ciro (2000). "El uso de los microdatos censales. Una aplicación a la migración interna en Colombia", Simposio de Estadistica 2000: Censos, Encuestas y Sistemas de Información Estadistica. Recuperado de: http://www.ced.uab.cat/publicacions/PapersPDF/Text186.pdf (3 de agosto de 2012).

Mincer, Jacob (1978). "Family Migration Decisions", The Journal of Political Economy, Vol. 86, No. 5, pp. 749-773.

Murad R., Rocío (2003). "Estudio sobre la distribución espacial de la población en Colombia”, Población y desarrollo, No. 48, pp. 1-68. Recuperado de: http://www.eclac.cl/cgi-bin/getProd.asp?xml=/publicaciones/ $\mathrm{xml} / 0 / 14000 / \mathrm{P} 14000 . \mathrm{xml} \& \mathrm{xsl}=/$ celade/tpl/p9f.xsl\&base=/tpl-i/topbottom.xslt (6 de agosto de 2012)

Pérez, José (2003). "Evidencia reciente del comportamiento de la migración interna en Colombia a partir de la Encuesta Continua de Hogares". 
Hernán Enríquez: Influencia de la migración en los precios de la vivienda...

Recuperado de: http://www.dane.gov.co/files/banco_datos/Migracion/ migracion_interna_Clbia.pdf (3 de agosto de 2012).

Potepan, Michael (1994). "Intermetropolitan Migration and Housing Prices Simultaneously determined?", Journal of Housing Economics, Vol. 3, Issue 2, pp. 77-91.

Potepan, Michael (1996). "Explaining Intermetropolitan variation in Housing Prices, Rents and Land Prices", Real Estate Economics, Vol. 24, Issue 2, pp. 219-245.

Poudyal, Neelam; Hodges, Donald G. \& Cordell, H. Ken (2008). “The role of natural resource amenities in attracting retirees: Implications for economic growth policy”, Ecological Economics, Vol. 68, Issue 1-2, pp. 240-248.

Roвacк, Jennifer (1982). "Wages, Rents, and the Quality of Life", Journal of Political Economy, Vol. 90, No. 6, pp. 1257-1278.

Romero, Jennifer (2010). "El éxito económico de los costeños en Bogotá: migración interna y capital humano", Documentos de Trabajo Sobre Economía Regional, No. 129, pp. 1-35. Recuperado de: http://www.banrep. gov.co/documentos/publicaciones/regional/documentos/DTSER-129. pdf (10 de agosto de 2012).

SJAASTAD, Larry (1962). "The Costs and Returns of Human Migration", The Journal of Political Economy, Vol. 70, No. 5, pp. 80-93.

Todaro, Michael P. (1969). "A Model of Labor Migration and Urban Unemployment in Less Developed Countries", The American Economic Review, Vol. 59, No. 1, pp. 138-148.

Waltert, Fabian \& Schläpfer, Felix (2010). "Landscape amenities and local development: A review of migration, regional economic and hedonic pricing studies", Ecological Economics, Vol. 70, Issue 2, pp. 141-152. 


\section{Anexo 1}

\section{Fuentes de datos}

\begin{tabular}{|c|c|c|}
\hline Nombre & Descripción & Fuente \\
\hline lnpvu & $\begin{array}{l}\text { Logaritmo natural del avalúo } \\
\text { de predios urbanos ajustado por } \\
\text { número de viviendas. }\end{array}$ & $\begin{array}{l}\text { Cálculos del autor a partir de infor- } \\
\text { mación del anuario Estadístico de Cun- } \\
\text { dinamarca } 2006\end{array}$ \\
\hline migneta & $\begin{array}{l}\text { Tasa de migración neta por cada } \\
100 \text { mil habitantes }\end{array}$ & $\begin{array}{l}\text { Cálculos del autor a partir de Censo } \\
2005 \text { Redatam }\end{array}$ \\
\hline logingpc & $\begin{array}{l}\text { Logaritmo natural de Ingresos } \\
\text { per cápita por Producto Interno } \\
\text { Bruto estimado }\end{array}$ & $\begin{array}{l}\text { Cálculos del autor a partir de Anuario Es- } \\
\text { tadístico de Cundinamarca } 2006\end{array}$ \\
\hline logdensidad & $\begin{array}{l}\text { Logaritmo natural de la po- } \\
\text { blación urbana dividida en el área } \\
\text { urbana en } \mathrm{km}^{2}\end{array}$ & $\begin{array}{l}\text { Cálculos del autor a partir de información } \\
\text { del anuario estadístico de Cundinamarca } \\
2006 \text { y la Federación Colombiana de } \\
\text { Municipios. }\end{array}$ \\
\hline rural & $\begin{array}{l}\text { Porcentaje del área rural respecto } \\
\text { al área total }\end{array}$ & $\begin{array}{l}\text { Cálculos del autor a partir de infor- } \\
\text { mación del anuario Estadístico de Cun- } \\
\text { dinamarca } 2006\end{array}$ \\
\hline logpred & $\begin{array}{l}\text { Logaritmo natural del impuesto } \\
\text { predial medio para la zona urbana }\end{array}$ & $\begin{array}{l}\text { Cálculos del autor a partir de Anuario Es- } \\
\text { tadístico de Cundinamarca } 2007\end{array}$ \\
\hline acces & $\begin{array}{l}\text { Índice de conectividad distancia } \\
\text { carretera en km dividido el número } \\
\text { de horas de duración del viaje a } \\
\text { Bogotá elevado al cuadrado. Nor- } \\
\text { malizado de } 0 \text { a } 100\end{array}$ & $\begin{array}{l}\text { Cálculos del autor a partir de la infor- } \\
\text { mación de Google Maps }\end{array}$ \\
\hline logles & $\begin{array}{l}\text { Logaritmo natural de la tasa de } \\
\text { lesiones interpersonales por cada } \\
100.000 \text { habitantes }\end{array}$ & $\begin{array}{l}\text { Cálculos del autor a partir de Anuario Es- } \\
\text { tadístico de Cundinamarca } 2006\end{array}$ \\
\hline Frio & $\begin{array}{l}\text { Variable dummy que toma valor } \\
1 \text { si el municipio se encuentra en } \\
\text { piso térmico Frio }\end{array}$ & Instituto Geográfico Agustín Codazzi \\
\hline desemp & Tasa de desempleo municipal & $\begin{array}{l}\text { Observatorio Laboral de Cundinamarca } \\
\text { con información de Redatam censo } 2005 .\end{array}$ \\
\hline
\end{tabular}


Hernán Enríquez: Influencia de la migración en los precios de la vivienda...

\section{Fuentes de datos}

\begin{tabular}{cll}
\hline Nombre & \multicolumn{1}{c}{ Descripción } & \multicolumn{1}{c}{ Fuente } \\
\hline lospershog & $\begin{array}{l}\text { Logaritmo natural del Ratio entre } \\
\text { el total de personas y el total de } \\
\text { hogares }\end{array}$ & $\begin{array}{l}\text { Cálculos del autor a partir de la infor- } \\
\text { mación disponible en el Anuario Es- } \\
\text { tadístico de Cundinamarca } 2006 .\end{array}$ \\
logpob014 & $\begin{array}{l}\text { logaritmo natural de la población } \\
\text { entre 0 a 14 ańos }\end{array}$ & $\begin{array}{l}\text { Cálculos del autor a partir de infor- } \\
\text { mación disponible en Anuario Estadísti- } \\
\text { co de Cundinamarca 2006 }\end{array}$ \\
& $\begin{array}{l}\text { \% de personas en el municipio con } \\
\text { nivel de escolaridad secundaria }\end{array}$ & DANE- Censo de 2005 \\
\hline
\end{tabular}

Fuente: elaboración propia.

\section{Anexo 2}

\begin{tabular}{lccccc}
\hline \multicolumn{5}{c}{ Estadística descriptiva Variables utilizadas } \\
\hline Variable & Obs. & Media & $\begin{array}{c}\text { Desviación Es- } \\
\text { tándar }\end{array}$ & Min & Max \\
lnpvu & 116 & 17,030 & 0,6167 & 15,724 & 19,689 \\
migneta & 116 & 20,667 & 11,903 & $-3,458$ & 65,053 \\
logingpc & 116 & 1,9787 & 0,4395 & 0,7394 & 3,7493 \\
logdensidad & 116 & 1,6061 & 0,8918 & $-3,218$ & 3,1535 \\
rural & 116 & 57,999 & 18,481 & 2,4857 & 93,063 \\
logpred & 116 & 5,5351 & 1,0163 & 2,7315 & 10,019 \\
acces & 116 & 31,0294 & 21,115 & 0 & 100 \\
logles & 116 & 5,7285 & 1,1157 & 2,4849 & 7,6048 \\
Frio & 116 & 0,7844 & 0,4129 & 0 & 1 \\
desemp & 116 & 20,666 & 2,0214 & 0,57 & 12,794 \\
logpershog & 116 & 1,2922 & 0,0941 & 1,0595 & 1,769 \\
logpob014 & 116 & 3,6833 & 0,3825 & 3,0013 & 4,91 \\
educ & 116 & 6,9183 & 0,9184 & 3,5989 & 9,79 \\
\hline
\end{tabular}

Fuente: elaboración propia a partir de distintas fuentes Ver Anexo 1. 
Anexo 3. Relación municipios migración precios de vivienda por grado expulsión - atracción

Ranking 5 municipios más receptores 2005

\begin{tabular}{clccc}
\hline No. & Municipio & $\begin{array}{c}\text { Migración neta } \\
\text { (personas) }\end{array}$ & $\begin{array}{c}\text { Avalúo medio viviendas } \\
\text { urbanas (pesos de 2005) }\end{array}$ & $\begin{array}{c}\text { Ingreso per cápita } \\
\text { (Millones de pesos de 2005) }\end{array}$ \\
\hline 5 & Girardot & 17.556 & 57.804 .527 & 6.563 \\
4 & Fusagasugá & 18.617 & 42.365 .892 & 4.484 \\
3 & Mosquera & 20.845 & 20.619 .335 & 7.535 \\
2 & Chía & 25.317 & 57.013 .866 & 5.044 \\
1 & Soacha & 173.175 & 22.175 .063 & 5.515
\end{tabular}

Ranking 5 municipios más expulsores 2005

\begin{tabular}{llccc}
\hline No. & Municipio & $\begin{array}{c}\text { Migración neta } \\
\text { (personas) }\end{array}$ & $\begin{array}{c}\text { Avalúo medio viviendas } \\
\text { urbanas (pesos de 2005) }\end{array}$ & $\begin{array}{c}\text { Ingreso per cápita } \\
\text { (Millones de pesos de 2005) }\end{array}$ \\
\hline 112 & Gama & 282 & 22.809 .393 & 42.502 \\
113 & Tibirita & 175 & 25.723 .747 & 11.097 \\
114 & Gutiérrez & -10 & 10.560 .345 & 6.627 \\
115 & Fómeque & -72 & 32.464 .899 & 7.502 \\
116 & Medina & -328 & 15.026 .188 & 9.234 \\
\hline
\end{tabular}

Fuente: elaboración propia a partir de Anuario Estadístico de Cundinamarca. 